\title{
POLYMERS: THE SECOND CENTURY
}

\author{
Gordana Bogoeva-Gaceva \\ Faculty of Technology and Metallurgy, Ss. Cyril and Methodius University, \\ Rudjer Bošković 16, 1000 Skopje, Republic of Macedonia \\ gordana@tmf.ukim.edu.mk
}

This year marks the $100^{\text {th }}$ anniversary of the revolutionary article published by the German chemist Hermann Staudinger (1881-1965), entitled "Über Polymerisation" (Ber. Dtsch. Chem. Ges. 53 (6) (1920) 1073-1085), which is considered as the beginning of macromolecular chemistry and polymer science in general.

In that article, Staudinger hypothesizes the chain structure of rubber molecules (and many other natural products, such as starch, cellulose and proteins), in which a large number of isoprene monomer units are covalently interconnected, thus building a huge molecule, much larger than previous convictions, calling it a macromolecule. Staudinger explained the unusual tensile strength and elasticity of rubber by the large length of the chain macromolecules and the numerous double bonds between the carbon atoms in the building blocks within each molecule.

At that time, most of his opponents, including many famous chemists like Wolfgang Oswald, Hans Pringsheim and Kurt Hess, supported the concept of cluster aggregates of small molecules that in some substances are joined together due to strong intermolecular aggregation bonds. Other leading chemists of the time, such as Carl Harries and Paul Karrer argued that natural materials such as rubber, cellulose, and proteins are made up of aggregates of small molecules that bind intermolecular bonds weaker than valence bonds, arguing that individual molecules with a relative mass greater than 5,000 must be unstable and that they would decompose spontaneously if synthesized. In contrast, Staudinger considered that in macromolecular compounds, in which units are linked by chemical bonds, molecular masses of few millions are possible [1, 2].

The debate, also known as the colloid/macromolecule debate, lasted a long time, and Staudinger strongly opposed the traditional analogy imposed between inorganic colloids and organic high-molecular compounds, which was defended by proponents of the colloid hypothesis.

After extensive experimental work and a very long period of controversy and denial, Staudinger's theory of macromolecules was finally accepted by the scientific community, and he won the Nobel Prize in Chemistry in 1953. At the award ceremony, these words will be remembered: "It is no secret that for a long time many colleagues rejected your views... Perhaps, this is understandable. In the world of polymers, almost everything was new and untested. Long standing established concepts had to be revised, or new ones created."

From 1920 until today, many important discoveries have taken place in the chemistry and physics of polymers, and polymers have evolved into a significant and expansive field of research that marked the twentieth century, simultaneously affecting many aspects of our lives.

We should not forget the road that led to the foundation of Studinger's discovery, which was long and thorny. Until 1930 he continued the experiments with chemically modified natural rubber, catalytically hydrogenated, in order to refine the effect of the double bond. The research was motivated by the claims of his opponents that the specific ("colloidal") properties of natural rubber are due to the double bonds in the constituent molecular units. Staudinger showed that the disappearance of the double bond, achieved by hydrogenation of the rubber, did not affect the "colloidal properties" of the material, and that, Staudinger believed, was the evidence of the existence of covalent bonds between the building blocks of rubber molecules. The results of these 
experiments were announced in 19 new published articles, which were again met with numerous criticisms and mistrust.

In addition to rubber, Staudinger's research on colloidal suspensions of various natural products in standard solvents is significant, as are the attempts to calculate the molecular weight of colloids from osmotic pressure measurements. The synthesis of polymers starting from styrene as a precursor, but also from other monomers, in Staudinger's laboratory resulted in homologous series, and the development of a complex correlation between viscosity and molecular mass of homologues (Staudinger's equation, which is further transformed into Mark-Houwink-Staudinger equation). It was concluded for the first time that not only the molecular mass, but also the geometry of the molecule had an impact on viscosity.

In the period 1920-1930, several important discoveries were made which were important for Staudinger's ideas, and in general - for polymer science.

In favor of Staudinger's macromolecular theory, were the researches of J. R. Katz, E. A. Hauser and H. Mark $(1925,1926)$ conducted by means of X-ray diffraction (at that time, a relatively new analytical technique) on tensed rubber, which indicated the possible presence of partially crystallized material. This discovery supported the existence of elastic macromolecules, which in the case of mechanical deformation can be arranged in oriented structures with a high degree of internal order, which resulted in the appearance of a characteristic X-ray image (patterns). At the same time, the first results were published on the structure of another natural polymer, cellulose, composed of chains of mutually covalently linked glycoside units (O. L. Sponsler, W. H. Dore). In 1924, thanks to the development of ultracentrifuge, Theodor Svedberg succeeded in accelerated sedimentation of some colloids, which enabled him to isolate pure substances, and estimating their molecular masses by comparing their sedimentation rates. Thus, Svedberg determined the relative mass of the hemoglobin molecule to be approximately 68,000 units, and that of haemocyanin - at about 5 million (1928).

It is interesting to note that Heinrich Wieland, winner of the Nobel Prize in Chemistry in 1927, wrote to Staudinger: "Dear colleague, drop the idea of large molecules; organic molecules with a molecular weight higher than $\mathbf{5 0 0 0}$ do not exist. Purify your products, such as rubber, then they will crystallize and prove to be low molecular compounds!"

Finally, the macromolecular nature of polymers was brilliantly confirmed by the syntheses of Wallace H. Carothers in DuPont Laboratories, when a new type of synthetic polymer was obtained - polyamide ("nylon"), which was melt-drawn into very strong and elastic fibers. In the condensation reaction between hexamethylene diamine and adipic acid, water was released as a by-product, by the amount of which the length of the macromolecules was estimated.

Thus begins the Golden Age of development of synthetic polymers, through the two basic mechanisms of synthesis - polymerization (addition polymerization) and polycondensation (stepwise polymerization), marked by mass production of such polymers as polyethylene, polypropylene, polyvinyl chloride, polyamides (aliphatic and aromatic), polyethylene terephthalate, polystyrene, polytetrafluoroethylene, polyurethanes, polyacrylates and many others. For the most widely used of them - great credit goes to the development of special catalytic systems for controlling the configuration of the macromolecular chain (stereo-regular polymerization), for which the Nobel Prize in Chemistry was awarded to Karl Ziegler and Giulio Natta in 1963. Another name of great importance for the development of polymer science must be mentioned - Paul J. Flory, winner of the Nobel Prize in Chemistry in 1974, whose research has shown that individual macromolecules of polymers are of different lengths, creating the basis for the term specific only for polymers - molecular mass distribution, which defines the physical, chemical, mechanical and other properties of polymers [3].

At the beginning of his speech on the occasion of the Nobel Prize ceremony, Staudinger said: "Macromolecular compounds include the most important substances occurring in nature such as proteins, enzymes, the nucleic acids, besides the polysaccharides such as cellulose, starch and pectins ... as well as rubber, and lastly the large number of new, fully synthetic plastics and artificial fibers. Macromolecular chemistry is very important both for technology and for biology." [4, 5].

Thanks to the gained knowledge of the specific structure of polymers and the exclusive properties that result from it, the twentieth century was marked by incredibly rapid development of methods for controlled synthesis of polymers, design of their structure and properties, development 
of hybrid materials, electrically conductive polymers, polymer composites and nanocomposites, advanced materials applied in electronics and energy, sustainable and environmentally friendly materials, biodegradable and biocompatible polymers, polymer materials for optical systems, etc. The application of polymers today covers many areas, from general purpose fibers in the textile industry to those with special properties, with a diameter of several tens of microns to nanometers, plastics for everyday use, functional materials, paints, varnishes and coatings - for general and special purpose (anti-corrosion, aesthetic, but also "intelligent", with adjustable properties depending on external factors), adhesives, materials for the automotive industry and construction, pharmacy and medicine, such as implants, for tissue engineering and drug carriers. If in the twentieth century organic polymers were dominant, both in terms of production volume and global economic effect, the twenty-first century is considered to be the century of inorganic polymers, with properties more advanced than many organic polymers and with new areas of possible applications. But the second century of polymers also faces a number of challenges related to the pollution of the environment with plastic waste and finding environmentally and economically optimal solutions for waste treatment, as well as to the growing concern for microplastics, which is already embedded in the life cycle of our planet.

It is interesting to note that Staudinger is the founder of the influential journal Macromolecular Chemie (now known as Macromolecular Chemistry and Physics). In honor of the jubilee, the first issue of the journals Macromolecular Chemistry and Physics and Macromolecular Rapid Communications for 2020 were available to all readers throughout the year.

The Section for Macromolecules, which is a member of the European Polymer Federation, has been functioning for decades within the Society of Chemists and Technologists of Macedonia (SHTM). The number of active members of the Section changes over the years, mainly due to the change in the structure of industrial and economic capacities in the country engaged in the production and processing of polymers, but it is important that its members continuously make their modest contribution to further development of polymer science through scientific and professional publications and domestic and international project collaboration.

\section{REFERENCES}

[1] H. Frey, T. Johann, Celebrating 100 years of "polymer science": Hermann Staudinger's 1920 manifesto, Polym. Chem. 11, 8 (2020). DOI: 10.1039/C9PY90161B

[2] Celebrating 100 years of polymer science. Progress in Polymer Science 100, 101193 (2020)

[3] Pioneers in Polymer Science, Raymond B. Seymour (Ed.), Kluwer Academic Publishers, 1989. DOI: 10.1007/978-94-009-2407-9

[4] The Nobel Prize in Chemistry. https://www.nobelprize.org/chemistry/laureates/1953/index.html

[5] https://www.nobelprize.org/nobel_prizes/chemistry/laureates/1953/staudinger-lecture.pdf 